\title{
miR-218 suppresses cardiac myxoma proliferation by targeting myocyte enhancer factor 2D
}

\author{
QUANXING CAO ${ }^{1}$, PINGSHUAN DONG ${ }^{2}$, YANYU WANG ${ }^{2}$, JUNWEI ZHANG ${ }^{1}$, \\ $\mathrm{XINGE} \mathrm{SHI}^{1}$ and YONGSHENG WANG ${ }^{1}$ \\ Departments of ${ }^{1}$ Cardiovascular Surgery and ${ }^{2}$ Cardiology, The First Affiliated Hospital of Henan University \\ of Science and Technology, Luoyang, Henan 471003, P.R. China \\ Received December 11, 2014; Accepted February 10, 2015
}

DOI: $10.3892 /$ or.2015.3861

\begin{abstract}
Cardiac myxoma is the most common type of human heart tumor, yet the molecular mechanism is still poorly understood. In the present study, we found that the level of myocyte enhancer factor 2D (MEF2D), a key regulatory protein for cardiac development, was elevated in specimens of cardiac myxoma, and was positively associated with the proliferation of myxoma cells. MEF2D suppression reduced the proliferation of myxoma cells and its tumorigenicity. Cell cycle progression was also inhibited by MEF2D suppression. miR-218, which is downregulated in myxoma, suppressed MEF2D expression by targeting its mRNA 3'UTR. Altogether, we found that miR-218/MEF2D may be an effective target for myxoma treatment.
\end{abstract}

\section{Introduction}

Cardiac myxoma is the most common type of heart tumor, and is associated with the risk of arterial embolism and sudden death (1). However, the molecular mechanism of myxoma initiation and progression remains unexplored. Interestingly, it has been well established that the genes associated with development always participate in the progression of cancer (2). Thus, the genes that are closely associated with heart development are worth studying for a better understanding of myxoma pathology.

The myocyte enhancer factor 2 (MEF2) family of transcription factors consists of 4 members in mammalian cells (MEF2A, 2B, 2C and 2D). They have been well documented to play important roles in the development of the heart (3). The expression levels of MEF2A, 2C and 2D were previously found to be elevated during cardiac development (4). Mice with mutation of MEF2C undergo looping morphogenesis failure

Correspondence to: Dr Pingshuan Dong, The Department of Cardiology, The First Affiliated Hospital of Henan University of Science and Technology, 24 Jinghua Road, Luoyang, Henan 471003, P.R. China

E-mail: pingshuandonghn@126.com

Key words: miR-218, MEF2D, myxoma during early cardiac morphogenesis (5). In Xenopus laevis, Mef2c and Mef2d both contribute to proper cardiac gene expression (6).

More importantly, the MEF2 family (particularly MEF2D) has been recently shown to be implicated in cancer biology. Studies concerning leukemia reveal that the fusion between $M E F 2 D$ and $D A Z A P 1$ caused by $\mathrm{t}(1 ; 19)(\mathrm{q} 23 ; \mathrm{p} 13.3)$ chromosome translocation contributes to the formation and progression of acute lymphoblastic leukemia (ALL) $(7,8)$. The role of MEF2D itself in cancer has also been reported in a recently published study. Ma et al provided evidence that MEF2D overexpression promotes the proliferation of liver cancer cells by suppressing cell cycle arrest-associated genes; and MEF2D overexpression may be due to the decline in tumor-suppressor miR-122 expression (9).

Notably, the association between MEF2D and heart disease has also been established in a study using a transgenic mouse model. MEF2D null mutation was able to prevent mice from stress-dependent cardiac hypertrophy, fetal gene activation and fibrosis, while the forced expression of MEF2D promoted the above pathological remodeling of the heart in mice (10). Atorvastatin was found to reverse this pathological modeling of the heart by suppressing the activity of MEF2D (11). These findings suggest that MEF2D is closely implicated in heart diseases.

However, the link between MEF2D and heart tumors has not yet been established. In the present study, we investigated the expression profile of the MEF2 family and their possible roles in cardiac myxoma. The underlying molecular mechanism was also investigated.

\section{Materials and methods}

Cell line culture. The human normal lung fibroblast cell line MRC-5, was purchased from the Shanghai Cell Collection (Shanghai, China). The cells were cultured in Dulbecco's modified Eagle's medium (DMEM) supplemented with $10 \%$ fetal bovine serum (FBS; Gibco-BRL) at $37^{\circ} \mathrm{C}$ under a humidified $5 \% \mathrm{CO}_{2}$ atmosphere.

Primary myxoma cell culture. The patient-derived primary cultures of cardiac myxoma cells were obtained from fresh tumor specimens from patients as previously described (12). 
Briefly, the single-cell suspension was obtained by mechanical manipulation of the cardiac myxoma specimen. The primary culture was maintained in DMEM supplemented with $10 \%$ FBS. The established primary myxoma cells were designated as CM001-CM006.

Quantitative PCR ( $P$ PCR) assay. The expression levels of mRNA of the MEF2 family and miR-218 were determined by qPCR. To examine the levels of MEF2A, MEF2C and MEF2D mRNA in cardiac myxoma, fresh tissues (cardiac myxoma samples and the matched non-cancerous tissue, $n=10$ ) were obtained with written informed consent from patients following the protocols approved by the Ethics Review Board of Henan University of Science and Technology (China). The patients underwent surgical operation at the Department of Cardiovascular Surgery, The First Affiliated Hospital of Henan University of Science and Technology. Total RNA was extracted with TRIzol solution (Sigma-Aldrich) according to the protocol provided by the manufacturer. The total RNA was transcribed into cDNAs using ReverTra Ace quantitative PCR (qPCR-RT) kit (Toyobo, Japan) according to the manufacturer's instructions. qPCR was performed using TaqMan ${ }^{\circledR}$ 2X Universal PCR Master Mix (Applied Biosystems) on a CFX96 ${ }^{\mathrm{TM}}$ Real-Time PCR Detection System (Bio-Rad Laboratories Inc., Hercules, CA, USA) supplied with analytical software. cDNA was obtained by PCR. The sequences of the used primers were previously described by Ma et al (9).

To detect the expression of miR-218, total RNA was extracted with TRIzol solution according to the protocols provided by the manufacturer. Reverse transcription reaction was carried out using All-in-One ${ }^{\mathrm{TM}}$ First-Strand cDNA Synthesis kit (AORT0020; GeneCopoeia) following the manufacturer's instructions. qPCR was performed using All-in-One ${ }^{\mathrm{TM}}$ miRNA qRT-PCR Detection kit (AOMD-Q020; GeneCopoeia) on a CFX96 ${ }^{\mathrm{TM}}$ Real-Time PCR Detection System supplied with analytical software. U6 was used as an endogenous reference. The primers and probes for miR-218 were purchased from GeneCopoeia (HmiRQP0327 and HmiRQP0326).

Immunoblotting assay. To examine the expression level of the indicated proteins, the lysate containing the total proteins extracted using M-PER ${ }^{\circledR}$ Mammalian Protein Extraction Reagent (Thermo Scientific, Rockford, IL, USA) was separated by polyacrylamide gel electrophoresis and transferred onto $0.45-\mu \mathrm{m}$ nitrocellulose membranes. After a 2-h blocking with $5 \%$ fat-free dry milk, the membranes were then incubated with primary antibodies for $2 \mathrm{~h}$. The membranes were incubated with the corresponding secondary antibody for $1 \mathrm{~h}$ and finally, visualized with SuperSignal West Dura Extended Duration Substrate (Thermo Scientific). The involved antibodies were all purchased from Cell Signaling Technology (Beverly, MA, USA).

MEF2D siRNA treatment. The MEF2D-specific siRNA was purchased from the Shanghai GenePharma Co. (Shanghai, China) as well as the control siRNA. The cells were transfected with the indicated siRNA $(50 \mathrm{nM}) 24 \mathrm{~h}$ prior to the subsequent experiments.

Proliferation assay. Primary cardiac myxoma cells $\left(5 \times 10^{3}\right)$ were planted into each well of 96-well plates. Overnight, the cells were treated under the indicated conditions. At the indicated time points, the cells were treated with $10 \mu \mathrm{l}$ 3-(4,5-dimethylthiazol-2-yl)-2,5-diphenyltetrazolium bromide (MTT) $(5 \mathrm{mg} / \mathrm{ml})$ for $4 \mathrm{~h}$. MTT was then removed, and $150 \mu \mathrm{l}$ DMSO was replaced. The spectrophotometric absorbance was detected on a Model 550 microplate reader at $570 \mathrm{~nm}$ with a reference wavelength of $655 \mathrm{~nm}$.

Animal experiments. All procedures for the animal experiments were approved by the Committee on the Use and Care on Animals in Henan University of Science and Technology and were performed following institutional guidelines. After the indicated treatments, human cardiac myxoma xenografts were established by subcutaneously inoculating $5 \times 10^{5}$ cells into both flanks of 5 -week-old BALB/c nude mice $(n=9)$. Tumor diameters were periodically measured with calipers. The volumes were calculated following the formula: Volume $\left(\mathrm{mm}^{3}\right)=$ length $(\mathrm{mm}) \mathrm{x}$ [width $\left.(\mathrm{mm})\right]^{2} / 2$. All animals received humane care according to the criteria outlined in the 'Guide for the Care and Use of Laboratory Animals' prepared by the National Academy.

Cell cycle analysis by flow cytometry. The cells were exposed to the indicated treatments, followed by being harvested, fixed in $70 \%$ ethanol and stained with propidium podide (PI; $200 \mathrm{mg} / \mathrm{ml}$ ) for flow cytometric analysis with the Aria II sorter (BD Biosciences). For each group, 10,000 cells were counted to determine the percentages of the $G_{0} / G_{1}, S$ and $G_{2} / M$ populations.

Identification of MEF $2 D$ as a target of miR-218. The potential evolutionarily conserved miR-218 targets were predicted using the algorithm TargetScan (http://www. targetscan.org/). Luciferase assay was applied to further confirm whether MEF2D is an authentic target of miR-218. To generate the luciferase reporter vectors, a 251-bp fully synthesized DNA construct identical to the region of the MEF2D 3'UTR containing the predicted miR-218 binding site (AAGCACA) was inserted into the SpeI and HindIII sites of the pMIR-REPORT vector (Applied Biosystems), generating pMIR-MEF2D-WT. To construct a control vector (pMIRMEF2D-MUT), a mutation in the miR-218 seed region was introduced into the Luc-m 3'UTR (AAGCTGA). The mimics of miR-218 were purchased from GenePharma Co. Plasmids for luciferase assays and mimics were cotransfected by Lipofectamine 2000 (Invitrogen) according to the protocols provided by the manufacturer. Subsequently, detection of luciferase activity was performed with the Dual-Luciferase ${ }^{\circledR}$ Reporter Assay kit (Promega, Madison, WI, USA) according to the manufacturer's instructions.

Statistical analysis. Each experiment was performed for at least three times. All values are reported as means $\pm \mathrm{SD}$, and compared at a given time point by the unpaired, two-tailed Student's test. Data were considered to indicate a statistically significant result at $\mathrm{P}<0.05$ and $\mathrm{P}<0.01$.

\section{Results}

MEF2D is overexpressed in cardiac myxoma. The expression profile of MEF2A, MEF2C and MEF2D mRNA 
A
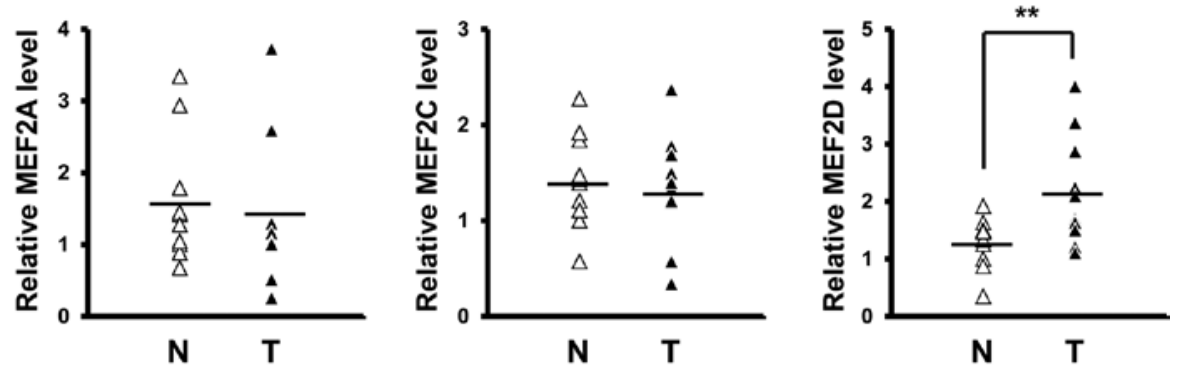

B

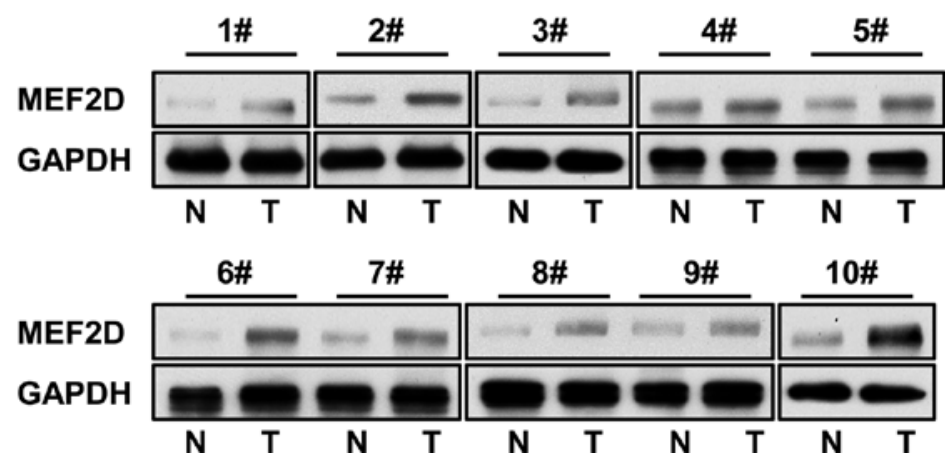

Figure 1. Aberrant expression profile of MEF2D in cardiac myxoma. (A) mRNA levels of MEF2A, MEF2C and MEF2D were examined in heart tumor specimens and their matched non-cancerous tissues $(n=10)$. Their expression levels were normalized by GAPDH. Data are shown as the mean \pm SD of three independent experiments. $\mathrm{T}$, cardiac myxoma; $\mathrm{N}$, non-cancerous tissue. ${ }^{* *} \mathrm{P}<0.01$. (B) The protein levels of MEF2D were determined by immunoblot analysis. GAPDH expression was used as an endogenous reference. T, cardiac myxoma; $\mathrm{N}$, non-cancerous tissue. MEF2D, myocyte enhancer factor 2D.

A
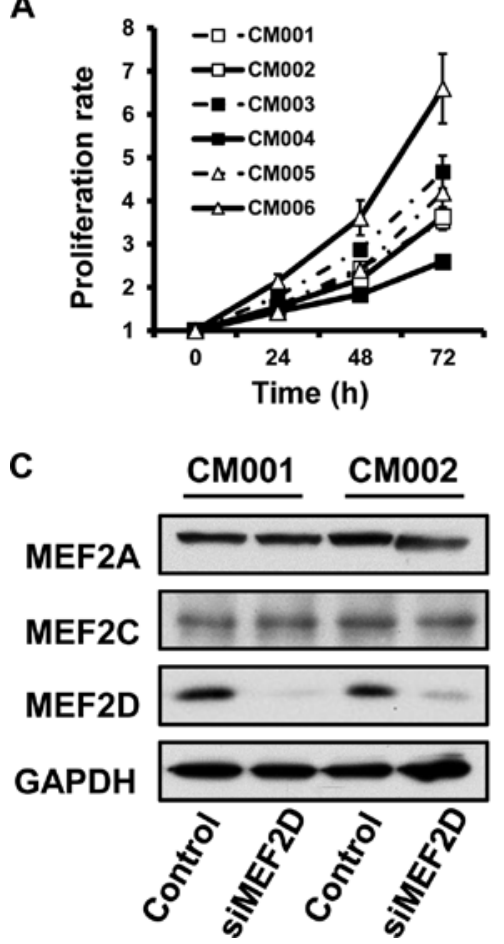

B
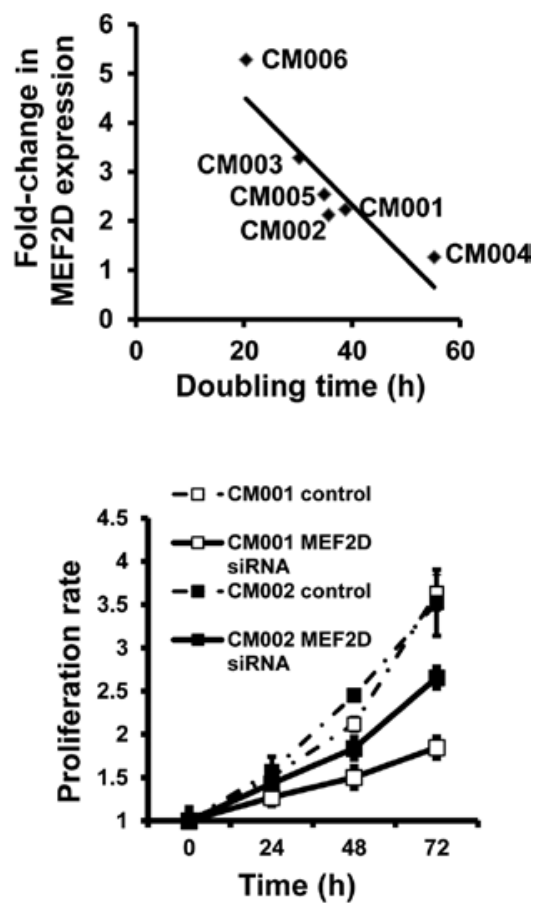

Figure 2. MEF2D overexpression contributes to the proliferation of cardiac myxoma cells. (A) The proliferation rates of patient-derived cardiac myxoma cells with different levels of MEF2D were determined by MTT assay. The data are shown as the mean \pm SD of three independent experiments. (B) The association between MEF2D levels and cell doubling time was also investigated in primary cardiac myxoma cells with Spearman analysis. (C) Cardiac myxoma cells were transfected with MEF2D siRNA or control (50 nM). Twenty-four hours later, MEF2A, MEF2C and MEF2D proteins were determined by immunoblot analysis. GAPDH served as an endogenous reference protein. (D) The proliferation rates of patient-derived cardiac myxoma cells transfected with MEF2D siRNA were determined by MTT assay. The data are shown as the mean $\pm \mathrm{SD}$ of three independent experiments. ${ }^{*} \mathrm{P}<0.05 ;{ }^{* * *} \mathrm{P}<0.01$. MEF2D, myocyte enhancer factor $2 \mathrm{D}$.

was investigated in fresh cardiac myxoma specimens and their matched non-cancerous tissues $(n=10)$. No significant difference was detected in the mRNA level of MEF2A and MEF2C between the cancer and normal tissues (Fig. 1A). 
A

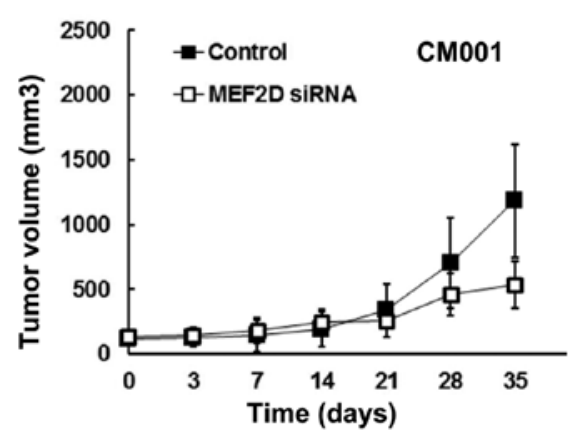

B

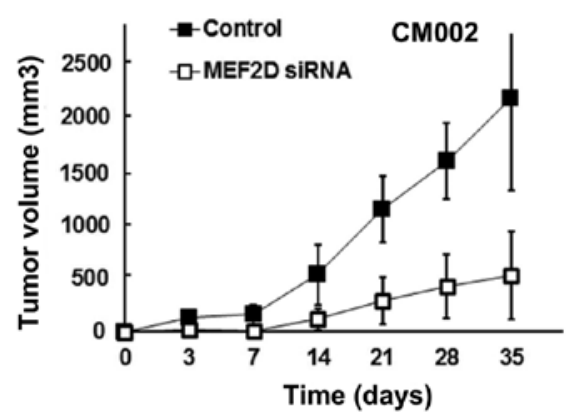

Figure 3. Downregulation of MEF2D expression impairs the tumorigenicity of cardiac myxoma cells. (A) CM001 and (B) CM002 cells were subcutaneously injected into the flanks of BALB/c nude mice after transfection with siMEF2D and control siRNA. The diameters of tumor were periodically measured and the volumes were calculated and shown. ${ }^{*} \mathrm{P}<0.05 ;{ }^{* *} \mathrm{P}<0.01$. MEF2D, myocyte enhancer factor $2 \mathrm{D}$.
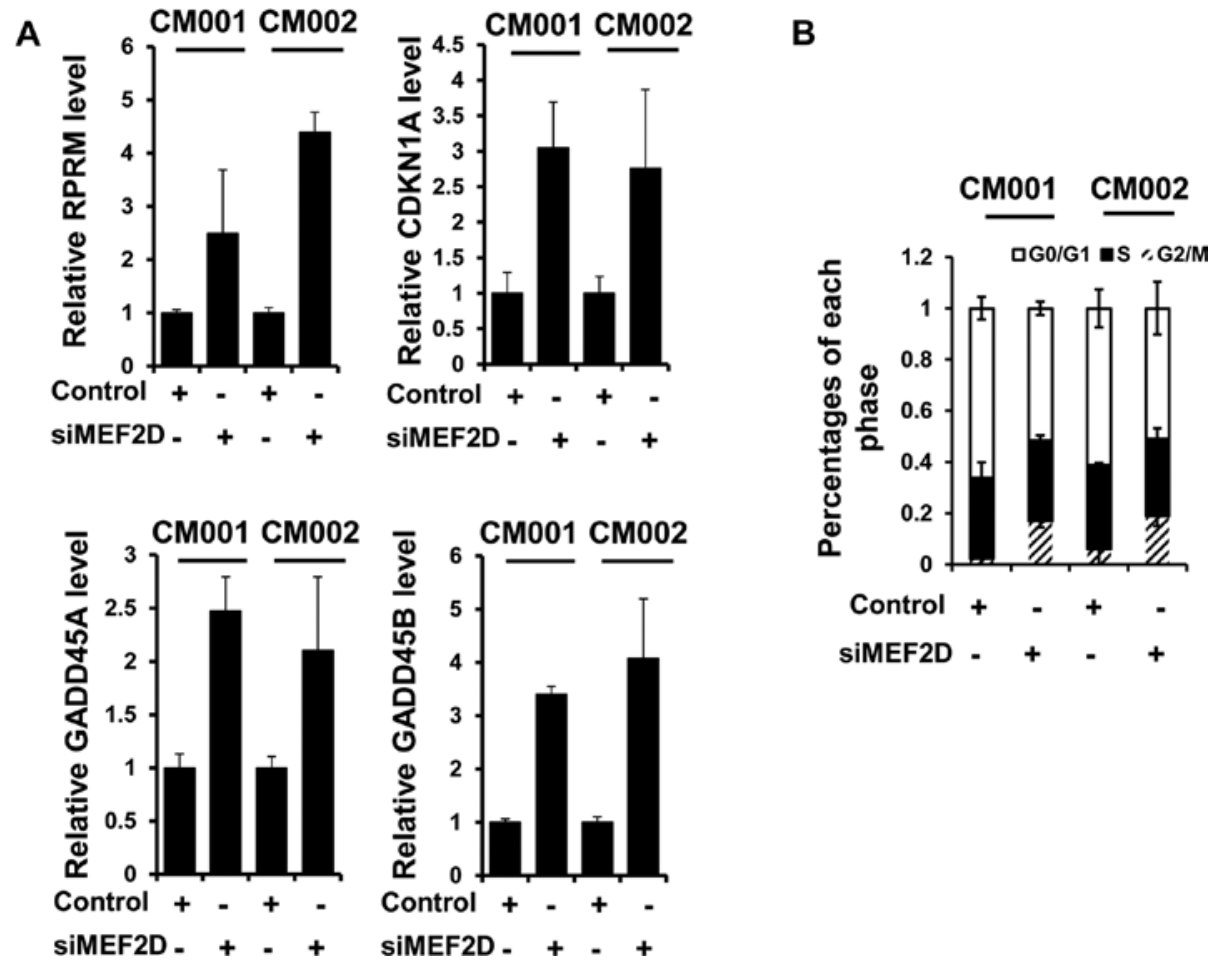

Figure 4. MEF2D facilitates cell cycle progression in cardiac myxoma cells. (A) The expression levels of RPRM, CDKN1A, GADD45A and GADD45B mRNA were evaluated in CM001 and CM002 cells transfected with MEF2D siRNA or control. The expression levels were normalized to GAPDH. Data are shown as the mean \pm SD of three independent experiments. (B) Cell cycle analysis was performed on CM001 and CM002 cells $48 \mathrm{~h}$ after transfection of MEF2D siRNA or control. The average values of the population percentages at $\mathrm{G}_{0} / \mathrm{G} 1, \mathrm{~S}$ and $\mathrm{G}_{2} / \mathrm{M}$ phases are shown as bars with $\pm \mathrm{SD}\left({ }^{*} \mathrm{P}<0.05 ;{ }^{* *} \mathrm{P}<0.01\right)$. MEF2D, myocyte enhancer factor 2D.

In contrast, MEF2D mRNA was found to be overexpressed in the cardiac myxoma specimens (Fig. 1A). Furthermore, immunoblot assay confirmed the differential expression of MEF2D between the cancer and normal tissues (Fig. 1B).

MEF2D expression is positively associated with the proliferation of cardiac myxoma cells. Given the role of MEF2D in the proliferation of hepatocellular carcinoma, we subsequently investigated whether aberrant expression of MEF2D is also associated with the proliferation of cardiac myxoma cells. MTT assays were employed to determine the proliferation rate of the primary myxoma cells. The data revealed that the levels of MEF2D protein were inversely correlated with the doubling time of the myxoma cells ( $\mathrm{R}=-0.943, \mathrm{P}=0.005)$ (Fig. 2A and B), revealing that cancer cells with higher MEF2D expression proliferated more rapidly.

$M E F 2 D$ suppression leads to the reduction in the proliferation rates of cardiac myxoma cells. We used small interfering RNA (siRNA) to specifically silence MEF2D expression, followed by investigation of the proliferation rate in myxoma cells. Our data showed that MEF2D siRNA selectively inhibited MEF2D expression, yet not MEF2A and MEF2C (Fig. 2C). We found that MEF2D downregulation inhibited the growth of the tested primary cardiac my xoma cells (Fig. 2D). Collectively, MEF2D overexpression promoted the proliferation of myxoma cells. 


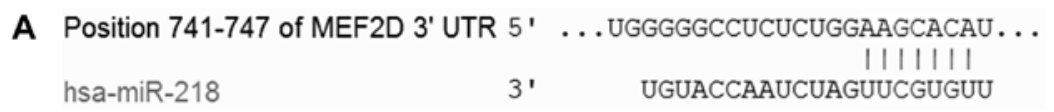

B

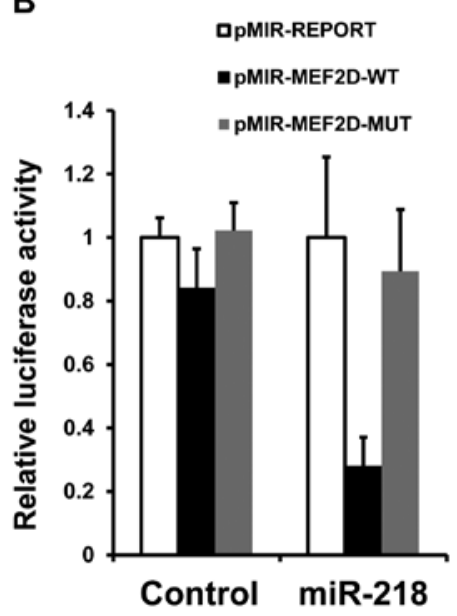

C

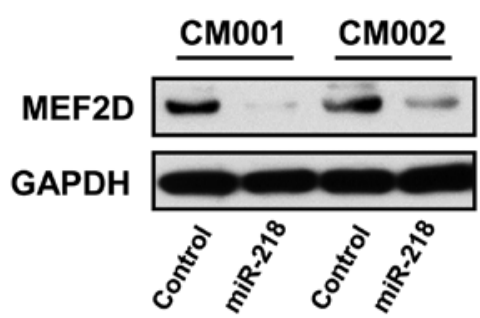

D

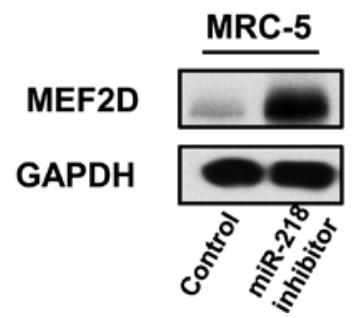

Figure 5. miR-218 suppresses MEF2D expression in cardiac myxoma cells. (A) A putative binding site targeted by miR-218 was predicted to be located in the 3'UTR of MEF2D mRNA. This site is highlighted in a grey box. (B) MRC-5 cells were co-transfected with miR-218 mimics or the control as well as luciferase reporter plasmids containing either wild-type (pMIR-MEF2D-WT) or mutant 3'UTR (pMIR-MEF2D-MUT) of the MEF2D gene. pMIR-REPORT was used as a control. Luciferase expression was measured $48 \mathrm{~h}$ after the above treatments. The data are shown as means \pm SD of three independent experiments. (C) MEF2D protein levels were detected in the CM001 and CM002 cells transfected with miR-218 mimics or the control. $\beta$-actin was used as a control. (D) MEF2D protein levels were detected in MRC-5 cells transfected with miR-218 inhibitor or control. $\beta$-actin was used as a control. MEF2D, myocyte enhancer factor 2D.

MEF2D downregulation impairs the tumorigenicity of cardiac myxoma cells. The effect of MEF2D overexpression on the tumorigenicity of myxoma cells was further studied in a mouse model. The tumors from the MEF2D siRNA-treated CM001 and CM002 cells were found to grow slower than the control groups by 71 and 65\%, respectively (Fig. 3A and B).

MEF2D facilitates cell cycle progression in cardiac myxoma cells. To elucidate the mechanisms underlying the promotion of proliferation and tumorigenesis of myxoma cells by MEF2D, we examined the expression of known MEF2D targets, RPRM, GADD45A, GADD45B and CDKN1A, following MEF2D siRNA treatment. Immunoblot analysis revealed that the expression levels of the above mRNAs were all increased when MEF2D expression was downregulated in the myxoma cells (Fig. 4A). Consistently, G2/M cell cycle arrest was also detected in both the CM001 and CM002 cells treated with MEF2D siRNA, evidenced by flow cytometric analysis (Fig. 4B). These results indicated that MEF2D contributed to the proliferation of myxoma cells by preventing $\mathrm{G} 2 / \mathrm{M}$ cell cycle arrest.

miR-218 suppresses MEF $2 D$ expression in myxoma cells by targeting the 3'UTR of its $m R N A$. The bioinformatic analysis showed that MEF2D is a predictive target of miR-218 using an algorithm available online (http://www.targetscan.org/) (Fig. 5A). Therefore, we employed luciferase assays to confirm whether miR-218 negatively regulates the expression of MEF2D by binding its putative recognition sites within 3'UTR of MEF2D mRNA. Luciferase expression by pMIR-MEF2DWT, but not pMIR-MEF2D-MUT, was shown to be greatly reduced in the myxoma cells treated with the miR-218 mimics (Fig. 5B). Furthermore, miR-218 overexpression was able to suppress the levels of endogenous MEF2D proteins in the CM001 and CM002 cells (Fig. 5C), while miR-218 suppression restored the expression of MEF2D in normal fibroblast MRC-5 cells (Fig. 5D). The above data indicated that MEF2D is an authentic miR-218 target.

miR-218 reduction promotes the proliferation of myxoma cells. In the same tumor specimens, miR-218 was found to be underexpressed (Fig. 6A). There was an inverse association between MEF2D mRNA and miR-218 levels $(\mathrm{R}=-0.881$, $\mathrm{P}=0001$ ) (Fig. 6B). MTT assays also demonstrated that miR-218 restoration was able to reduce the proliferation of the myxoma cells (Fig. 6C). These data indicate that downregulation of miR-218 could be responsible for the elevated expression of MEF2D and increased proliferation rates in the myxoma cells.

\section{Discussion}

For the first time, the present study provides evidence that development-associated regulators, MEF2 family proteins, are implicated in cardiac myxoma. Specifically, MEF2D, yet not other members, was shown to be differentially expressed in the heart tumor tissues. Generally, the etiology of sporadic cardiac myxomas remains elusive. Sauls et al reported that valve interstitial cells regulate matrix organization during foetal valve development through a serotonin, TG and filaminA pathway. Disrupting these key regulatory interactions can set the stage for the generation of postnatal myxomatous valve disease (13). For familial (but not sporadic) cardiac myxomas, 


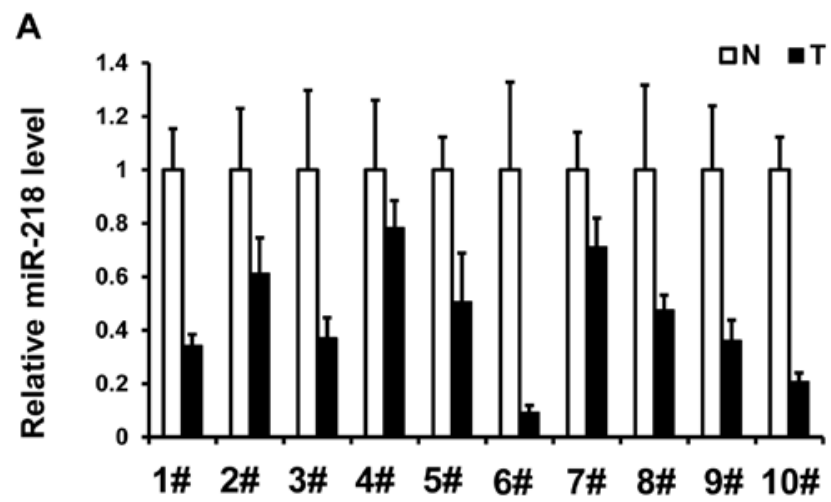

B

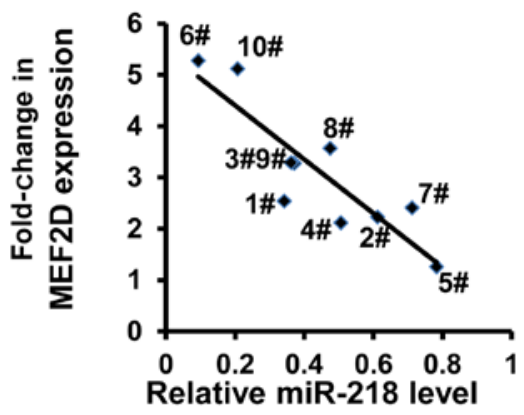

C

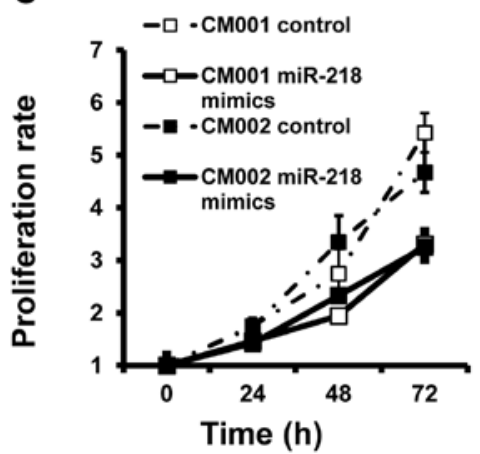

Figure 6. miR-218 suppression promotes the proliferation of myxoma cells. (A) miR-218 expression was examined in heart tumor specimens and their matched non-cancerous tissues $(n=10)$. The expression levels were normalized to GAPDH. Data are shown as the mean \pm SD of three independent experiments. T, cardiac myxoma; N, non-cancerous tissue. ${ }^{*} \mathrm{P}<0.05 ;{ }^{* *} \mathrm{P}<0.01$. (B) The association between miR-218 and MEF2D levels was determined by Spearman analysis in the cardiac myxoma specimens. (C) Cardiac myxoma cells were transfected with miR-218 mimics or control (50 nM). Then, the proliferation rates were determined by MTT assay. The data are shown as the mean $\pm \mathrm{SD}$ of three independent experiments. ${ }^{*} \mathrm{P}<0.05$; ${ }^{* *} \mathrm{P}<0.01$. MEF2D, myocyte enhancer factor $2 \mathrm{D}$.

germ-line mutations in the PRKAR1A gene were found to be associated with this type of heart neoplasm $(14,15)$. However, the molecular mechanism of myxomagenesis is still far from being completely elucidated.

The oncogenic role of MEF2D has been well established in liver cancer and leukemia. The involved mechanism appears to be primarily focused on the accelerated proliferation of these cancerous cells $(8,9)$. Our data further confirmed these previous findings, since MEF2D suppression reduced cell proliferation, impaired tumorigenicity, and induced cell cycle arrest at the $\mathrm{G}_{2} / \mathrm{M}$ phase. Notably, MEF2D overexpression was found to mediate stress-induced heart hypertrophy (10), implying this transcriptional factor may also facilitate the proliferation of normal cardiac muscle cells.

In addition, we confirmed that miR-218, a tumor suppressor in many types of cancers (16-18), suppressed the expression of MEF2D mRNA by targeting its 3'UTR. In fact, miRNAs have been verified as major regulators of MEF2D expression. miR-92 was shown to be overexpressed in hippocampal neurons induced by fear and to downregulate the expression of MEF2D (19). miR-122, a potent liver cancer suppressor, was also identified as a negative regulator of MEF2D expression (9).

Although miRNAs have been found to be closely associated with the progression of various cancers, the link between miRNAs and cardiac myxoma remains unexplored. Our study provides evidence that miRNAs can also participate in the growth of heart tumors, in line with their roles in other types of tumors.
Collectively, the present study provides evidence that MEF2D promotes the proliferation of human cardiac myxoma cells. Our data also demonstrated that the miR-218/MEF2D pathway may be an effective therapeutic target for patients with myxoma.

\section{References}

1. Tatli S and Lipton MJ: CT for intracardiac thrombi and tumors. Int J Cardiovasc Imaging 21: 115-131, 2005.

2. Rubin P, Williams JP, Devesa SS, Travis LB and Constine LS: Cancer genesis across the age spectrum: Associations with tissue development, maintenance, and senescence. Semin Radiat Oncol 20: 3-11, 2010.

3. Rana MS, Christoffels VM and Moorman AF: A molecular and genetic outline of cardiac morphogenesis. Acta Physiol 207: 588-615, 2013

4. Edmondson DG, Lyons GE, Martin JF and Olson EN: Mef2 gene expression marks the cardiac and skeletal muscle lineages during mouse embryogenesis. Development 120: 1251-1263, 1994.

5. Lin Q, Schwarz J, Bucana C and Olson EN: Control of mouse cardiac morphogenesis and myogenesis by transcription factor MEF2C. Science 276: 1404-1407, 1997.

6. Guo Y, Kühl SJ, Pfister AS, Cizelsky W, Denk S, Beer-Molz L and Kühl M: Comparative analysis reveals distinct and overlapping functions of Mef2c and Mef2d during cardiogenesis in Xenopus laevis. PLoS One 9: e87294, 2014.

7. Prima V, Gore L, Caires A, Boomer T, Yoshinari M, Imaizumi M, Varella-Garcia M and Hunger SP: Cloning and functional characterization of MEF2D/DAZAP1 and DAZAP1/MEF2D fusion proteins created by a variant $\mathrm{t}(1 ; 19)(\mathrm{q} 23 ; \mathrm{p} 13.3)$ in acute lymphoblastic leukemia. Leukemia 19: 806-813, 2005.

8. Prima V and Hunger SP: Cooperative transformation by MEF2D/ DAZAP1 and DAZAP1/MEF2D fusion proteins generated by the variant $t(1 ; 19)$ in acute lymphoblastic leukemia. Leukemia 21 : 2470-2475, 2007 
9. Ma L, Liu J, Liu L, et al: Overexpression of the transcription factor MEF2D in hepatocellular carcinoma sustains malignant character by suppressing $\mathrm{G}_{2}-\mathrm{M}$ transition genes. Cancer Res 74 : 1452-1462, 2014.

10. Kim Y, Phan D, van Rooij E, Wang DZ, McAnally J, Qi X, Richardson JA, Hill JA, Bassel-Duby R and Olson EN: The MEF2D transcription factor mediates stress-dependent cardiac remodeling in mice. J Clin Invest 118: 124-132, 2008.

11. Geng J, Zhao Z, Kang W, Wang W, Zhang Y and Zhiming GE: Atorvastatin reverses cardiac remodeling possibly through regulation of protein kinase $\mathrm{D} /$ myocyte enhancer factor $2 \mathrm{D}$ activation in spontaneously hypertensive rats. Pharmacol Res 61: 40-47, 2010.

12. Negishi M, Sakamoto H, Sakamaki T, Ishikawa O, Kanda T, Tamura J, Kurabayashi M and Nagai R: Disaccharide analysis of glycosaminoglycans synthesized by cardiac myxoma cells in tumor tissues and in cell culture. Life Sci 73: 849-856, 2003.

13. Sauls K, de Vlaming A, Harris BS, et al: Developmental basis for filamin-A-associated myxomatous mitral valve disease. Cardiovasc Res 96: 109-119, 2012.

14. Mantovani G, Bondioni S, Corbetta S, et al: Analysis of GNAS1 and PRKAR1A gene mutations in human cardiac myxomas not associated with multiple endocrine disorders. J Endocrinol Invest 32: 501-504, 2009.
15. Yin Z, Jones GN, Towns WH II, Zhang X, Abel ED, Binkley PF, Jarjoura D and Kirschner LS: Heart-specific ablation of Prkarla causes failure of heart development and myxomagenesis. Circulation 117: 1414-1422, 2008

16. Gao X and Jin W: The emerging role of tumor-suppressive microRNA-218 in targeting glioblastoma stemness. Cancer Lett 353: 25-31, 2014.

17. Wei Y, Du Y, Chen X, Li P, Wang Y, Zang W, Zhao L, Li Z and Zhao G: Expression patterns of microRNA-218 and its potential functions by targeting CIP2A and BMI1 genes in melanoma. Tumour Biol 35: 8007-8015, 2014.

18. Zhang C, Ge S, Hu C, Yang N and Zhang J: miRNA-218, a new regulator of $\mathrm{HMGB} 1$, suppresses cell migration and invasion in non-small cell lung cancer. Acta Biochim Biophys Sin 45: 1055-1061, 2013.

19. Vetere G, Barbato C, Pezzola S, Frisone P, Aceti M, Ciotti M, Cogoni C, Ammassari-Teule M and Ruberti F: Selective inhibition of miR-92 in hippocampal neurons alters contextual fear memory. Hippocampus 24: 1458-1465, 2014. 